\title{
A survey of antibodies to pestivirus in sheep in the Republic of Ireland
}

Ronan G. O’Neill, Michael O'Connor and Patrick J. O'Reilly

Department of Agriculture and Food, Central Veterinary Research Laboratory, Abbotstown, Castleknock, Dublin 15, Ireland.

Sera from 1,448 adult ewes in 91 flocks, representing all 26 counties in the Republic of Ireland, were examined for pestivirus antibodies using a commercially available ELISA which detected $\mathrm{IgG}_{1}$ antibody to border disease virus. Eighty-one sheep (5.6\%) in 42 flocks $(46.0 \%)$ were antibody-positive. Within infected flocks, the mean seroprevalence level was $11.4 \%$ with a range of $6.3 \%$ to $30.0 \%$. The highest antibody prevalence was detected in sheep from central lowland counties of Ireland. Comparative neutralisation testing of 42 ELISA-positive sera detected geometric mean antibody titres of 136 to the NADL strain of bovine viral diarrhoea virus (BVDV), 92 to the Moredun strain of border disease virus and 21 to the $137 / 4$ strain of border disease virus. These results suggest that BVDV may be the major ruminant pestivirus infecting sheep in Ireland. Although there are high numbers of infected flocks, many sheep within such flocks remain antibody-negative and are at risk of giving birth to lambs with congenital border disease.

\section{Key words}

Sheep,

Pestivirus, Border disease, Republic of Ireland, Antibody

Irish Veterinary Journal

Volume 57: 525 - 530, 2004

\section{Introduction}

The primary members of the genus Pestivirus are classical swine fever virus (CSFV), bovine viral diarrhoea virus (BVDV) types I and II, and border disease virus (BDV). CSFV is host specific under natural circumstances (i.e., it infects pigs only); BDV affects sheep, goats and pigs; and BVDV genotypes I and II affect cattle, sheep, goats, deer and pigs (Hurtado et al., 2003). Consequently, current recommendations are that the virus genome, rather than species of origin, be used as the basis for a revised genus classification (Vilcek et al., 1999).

Border disease virus spreads naturally by horizontal and vertical transmission. Oro-nasal infection in healthy adults or neonates

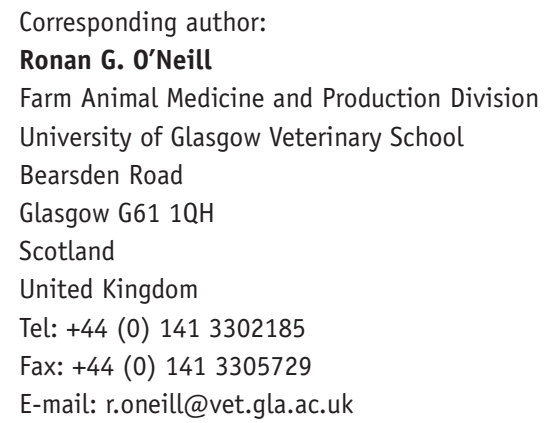

causes mild or inapparent disease. The consequences of infection are primarily reproductive - barren ewes, abortions, stillbirths and stunted, weak lambs with variable degrees of nervous dysfunction (Nettleton et al., 1998; Pratelli et al., 1999). Other occasional effects include 'hairy' and malpigmented wool, skeletal abnormalities, and immunosuppression with subsequent secondary bacterial infection (Jeffrey and Roeder, 1987; Pratelli et al., 1999). However, border disease is an uncommon clinical finding, in contrast to the pervasive consequences of BVDV infection in the cattle population in the Republic of Ireland (Gunn, 1996). When they do occur, outbreaks of clinical BDV can significantly

\begin{tabular}{|ll|}
\hline \multicolumn{2}{l|}{ Abbreviations } \\
BDV & Border disease virus \\
BVDV & Bovine viral diarrhoea virus \\
CI $_{95}$ & $95 \%$ confidence interval \\
COD & Corrected optical density \\
CSFV & Classical swine fever virus \\
ELISA & Enzyme-linked immunosorbent assay \\
FBK & Foetal bovine kidney \\
FMD & Foot-and-mouth disease \\
HRPO & Horseradish peroxidase \\
NI & Northern Ireland \\
SNT & Serum neutralisation test \\
\hline
\end{tabular}




\begin{tabular}{lccccc} 
Flock size & $\mathbf{0}-\mathbf{4 9}$ & $\mathbf{5 0}-\mathbf{9 9}$ & $\mathbf{1 0 0}-\mathbf{1 9 9}$ & $\mathbf{2 0 0}-\mathbf{2 9 9}$ & $\mathbf{3 0 0 +}$ \\
\hline No. of flocks & 11 & 20 & 36 & 16 & 8 \\
\hline No. of different counties & 7 & 14 & 18 & 12 & 6
\end{tabular}

reduce productivity in individual flocks (Bonniwell et al., 1987). Genomic, antigenic and serological studies have shown that BDV is more closely related to CSFV than to BVDV (Vilcek et al., 1997). BDV infection of pigs complicates the serological diagnosis of CSFV, a Class A notifiable disease (Pearson, 1992), and is a potential cause of antibody false-positives with serious implications for animal movement and trade. In addition, congenital infection of pigs with BDV can mimic clinical infection with low virulence strains of CSFV (Paton and Done, 1994).

The objectives of the present study were to assess the serological prevalence of border disease virus in the Irish sheep population, and to explore the relationship and cross-reactions between antibodies to BDV and the other important pestivirus, BVDV. Similar studies have been performed in the UK (Sands and Harkness, 1978), Norway (Loken et al., 1991), Sweden (Lunden et al., 1992), Denmark (Tegtmeier et al., 2000) and Northern Ireland (Graham et al., 2001).

\section{Materials and methods}

\section{Animals}

Sera from 1,448 adult ewes, collected from 91 flocks, were examined by ELISA for BDV antibodies. Flocks were tested from every county in the Republic of Ireland, the number selected for each county was weighted to represent the respective proportion of the national flock farmed there according to the 2001 National Sheep Census figures (Costelloe et al., 2002). The flocks selected had a mean size of 172; the largest had 744 sheep and the smallest had 18 sheep. Table 1 shows how these sheep flocks were distributed, both by size and by county.

Sixteen sera per flock were tested with the exception of one flock in Co Kildare, with eight sera only. The blood samples were collected as part of the surveillance for foot-and-mouth disease (FMD) virus antibodies over a six-week period: May 17, 2001 to June 26, 2001. At this time serum was collected, centrifuged and stored at $-20^{\circ} \mathrm{C}$. Eight nonadjacent flocks were identified as having high seroprevalence to pestivirus $(\geq 20 \%)$ and underwent a further targeted round of testing, using all available sera.

\section{Tests}

Border disease ELISA

A commercially available solid-phase indirect ELISA (BDV-Ab, SVANOVA Biotech, Sweden) was used according to the manufacturer's instructions. Results were read at $450 \mathrm{~nm}$. Corrected optical density (COD) values of less than 0.25 were regarded as negative.

\section{TABLE 2: Numbers and percentages of flocks and individual sheep seropositive to pestivirus by ELISA per county in the Republic of Ireland}

\begin{tabular}{lccccccc} 
County & $\begin{array}{c}\text { Number } \\
\text { of flocks }\end{array}$ & $\begin{array}{c}\text { Number } \\
\text { of sheep } \\
\text { tested }\end{array}$ & $\begin{array}{c}\text { Number } \\
\text { tested }\end{array}$ & $\begin{array}{c}\text { positive } \\
\text { Flocks }\end{array}$ & $\begin{array}{c}\text { Sheep } \\
\text { positive }\end{array}$ \\
\hline Carlow & 3 & 48 & 3 & 4 & 100 & 8 \\
\hline Cavan & 2 & 32 & 2 & 4 & 100 & 13 \\
\hline Clare & 1 & 16 & 1 & 2 & 100 & 13 \\
\hline Cork & 5 & 80 & 3 & 7 & 60 & 9 \\
\hline Donegal & 8 & 128 & 3 & 3 & 38 & 2 \\
\hline Dublin & 1 & 16 & 0 & 0 & 0 & 0 \\
\hline Galway & 10 & 160 & 4 & 8 & 40 & 5 \\
\hline Kerry & 7 & 112 & 3 & 4 & 43 & 4 \\
\hline Kildare & 3 & 40 & 3 & 3 & 100 & 8 \\
\hline Kilkenny & 3 & 48 & 1 & 1 & 33 & 2 \\
\hline Laois & 3 & 48 & 0 & 0 & 0 & 0 \\
\hline Leitrim & 2 & 32 & 0 & 0 & 0 & 0 \\
\hline Limerick & 1 & 16 & 0 & 0 & 0 & 0 \\
\hline Longford & 2 & 32 & 1 & 2 & 50 & 6 \\
\hline Louth & 2 & 32 & 1 & 1 & 50 & 3 \\
\hline Mayo & 7 & 112 & 0 & 0 & 0 & 0 \\
\hline Meath & 3 & 48 & 3 & 11 & 100 & 23 \\
\hline Monaghan & 2 & 32 & 1 & 6 & 50 & 19 \\
\hline Offaly & 3 & 48 & 3 & 11 & 100 & 23 \\
\hline Roscommon & 4 & 64 & 3 & 4 & 75 & 6 \\
\hline Sligo & 2 & 32 & 0 & 0 & 0 & 0 \\
\hline Tipperary & 4 & 64 & 2 & 3 & 50 & 5 \\
\hline Waterford & 2 & 32 & 1 & 2 & 50 & 6 \\
\hline Westmeath & 2 & 32 & 1 & 1 & 50 & 3 \\
\hline Wexford & 4 & 64 & 1 & 1 & 25 & 2 \\
\hline Wicklow & 5 & 80 & 2 & 3 & 40 & 4 \\
\hline Totals & 91 & 1448 & 42 & 81 & & \\
\hline & & & & & & \\
\hline
\end{tabular}

Bovine viral diarrhoea virus serum neutralisation test

The BVDV serum neutralisation test (SNT), using the cytopathic NADL strain (Hamilton and Timoney, 1973), was performed as described previously (Lenihan and Collery, 1977) on 42 BDV-ELISA-positive sera as identified above, at dilutions $1 / 8$ to $1 / 1,024$.

Border disease serum neutralisation tests

Serum neutralisation tests, using the Moredun strain of BDV, were performed in a sheep thymus cell line, SFT-R, while tests 


\section{TABLE 3: Results of comparative serum neutralisation testing of $\mathbf{4 2}$ ovine sera that were seropositive on screening using pestivirus-specific ELISA}

\begin{tabular}{lcc}
$\begin{array}{l}\text { Virus strain } \\
\text { (pestivirus } \\
\text { designation) }\end{array}$ & $\begin{array}{c}\text { No. of } \\
\text { positive sera } \\
(\geq 1 / 8)\end{array}$ & $\begin{array}{c}\text { Reciprocal } \\
\text { geometric mean } \\
\text { antibody titre }\end{array}$ \\
\hline NADL (BVDV) & 39 & 136 \\
\hline Moredun (BDV) & 39 & 92 \\
\hline $137 / 4(B D V)$ & 36 & 21 \\
\hline
\end{tabular}

with the $137 / 4 \mathrm{BDV}$ strain were performed using secondary foetal bovine kidney (FBK) cell cultures. Sera were tested over a dilution range of $1 / 8$ to $1 / 1,024$. After incubation for three days, the monolayers were fixed at $80^{\circ} \mathrm{C}$, overlaid with porcine polyclonal pestivirus antiserum followed by a commercial HRPO-conjugated rabbit anti-pig immunoglobulin. Virus growth was indicated by the presence of reddish brown intracytoplasmic staining on microscopic examination (Jensen, 1981).

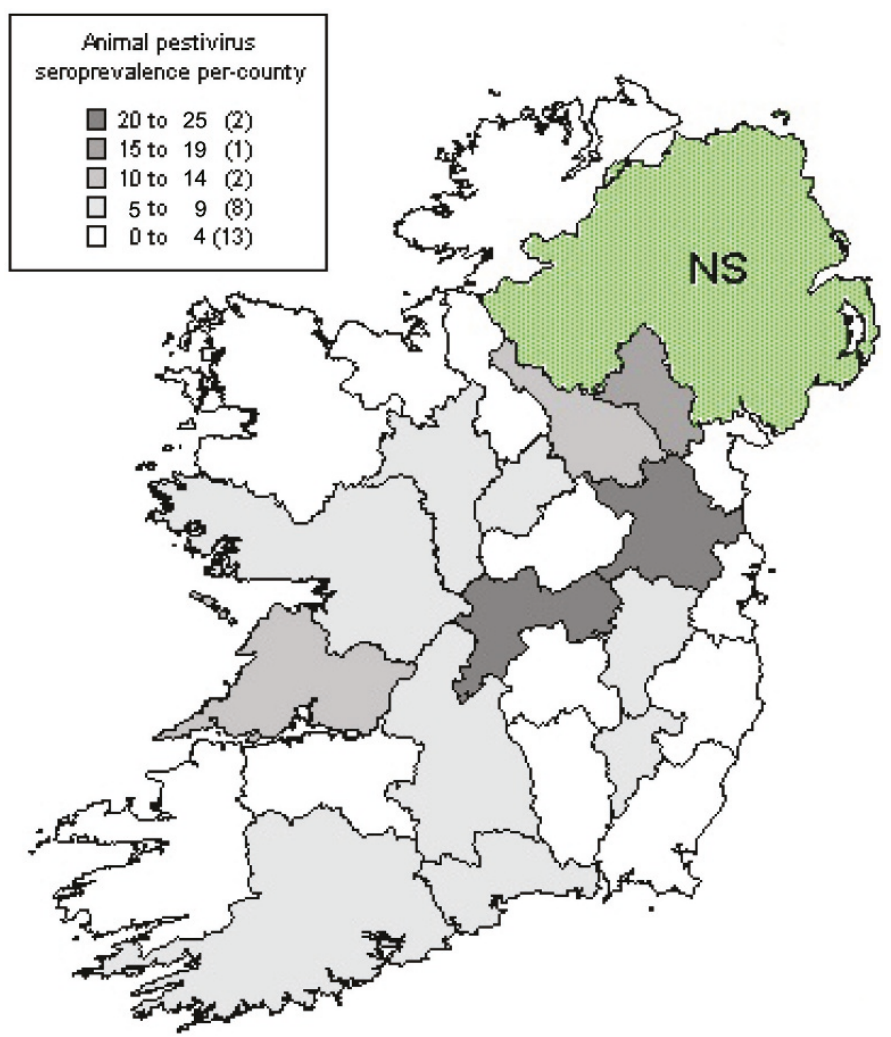

FIGURE I: Percentage of individual sheep that were seropositive to pestivirus in each county in the Republic of Ireland. NS: not sampled.

\section{Results}

A total of $81\left(5.6 \%, \mathrm{CI}_{95} \pm 1.2 \%\right)$ of the 1,448 sheep were positive for pestivirus antibody. Positive sheep were detected in $42\left(46 \%, \mathrm{CI}_{95} \pm 10.2 \%\right)$ of the 91 sheep flocks tested (Table 2$)$. The average pestivirus antibody prevalence among the positiveonly flocks was $11.4 \%$, with a maximum of $30.0 \%$ and a minimum $6.3 \%$. The mean COD of the positive sera was 0.59 , with a minimum of 0.25 and a maximum of 1.39 .

The second focused phase of testing concentrated on 139 samples taken from eight nonadjacent flocks identified as having high seroprevalence. Twenty-eight $(20.1 \%)$ of these second phase samples proved positive. The mean overall intraflock antibody prevalence among this specific group of eight flocks was $23.9 \%\left(\mathrm{CI}_{95} \pm 5.1 \%\right)$ with a range of $15.7 \%$ to $30.0 \%$; the mean positive COD among this subset of samples remained virtually unchanged at 0.61 .

The per-county geographic pattern of seroprevalence among individual animals and among individual flocks is illustrated in Figures 1 and 2 .

The results of testing 42 pestivirus-antibody ELISA-positive sera by comparative neutralisation tests are shown in Table 3 . Thirty-nine sera contained neutralising antibody to the BVDV

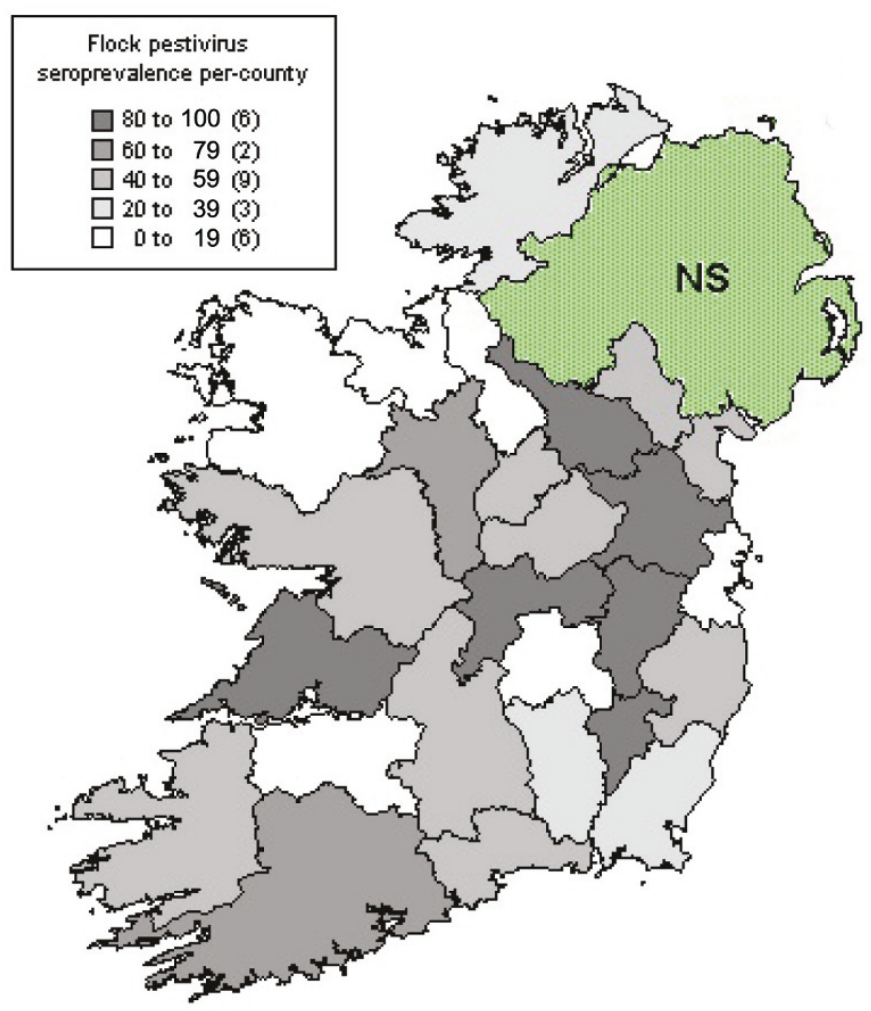

FIGURE 2: Percentage of sheep flocks that were seropositive to pestivirus in each county in the Republic of Ireland. NS: not sampled. 
strain, NADL and the BDV strain, Moredun, while 36 sera contained neutralising antibody to BDV strain $137 / 4$. Three ELISA-positive sera (mean COD $=0.29$ ) had neutralising titres less than $1 \frac{1}{8}$ in all SNTs. The highest geometric mean antibody titres were obtained against the NADL strain of BVDV followed by the Moredun strain, with strain 137/4 having lowest geometric mean antibody titres. Among those that tested positive to the NADL strain, the reciprocal geometric mean titre was 136 , with a minimum of 16 and a maximum of 512 . For the other SNTs, Moredun reciprocal antibody titres ranged between 8 and 1,024, while strain $137 / 4$ antibody titres were limited to between 8 and 64 . Thirty-four $(81 \%)$ sera had fourfold or greater ( $\geq 2$ dilutions) antibody titres to BVDV than to BDV strain $137 / 4$, while $16(38 \%)$ sera had four-fold or greater antibody titres to BVDV than to BDV strain Moredun. Higher antibody titres to BDV strain $137 / 4$ compared to BVDV were not detected, although three sera had four-fold or greater antibody titres to BDV strain Moredun than to BVDV.

\section{Discussion}

The level of seroprevalence for pestivirus antibodies found in the initial phase of this survey approximated well with the 5.3\% found in a study of the Northern Ireland (NI) flock (Graham $e t$ al., 2001). As all sampled sheep were of breeding age, it was assumed that maternal antibodies no longer persisted and that antibody indicated direct exposure to pestivirus. The detected seroprevalence may underestimate pestivirus activity in infected flocks. While the antibody has persisted for up to 485 days in ewes, infected when pregnant (Huck et al., 1975), it may become undetectable earlier in other sheep (Sawyer et al., 1986). Out of the 91 flocks tested, $42(46 \%)$ were found to contain sheep that were seropositive for pestivirus. This flock seroprevalence level was considerably higher than the $30.4 \%$ reported in the NI study by Graham et al. (2001) and the $32.6 \%$ flock prevalence for indigenous sheep reported in an earlier NI study (Adair et al., 1984). This disparity may be because a distinction was not made in the present survey for flocks with higher numbers of non-indigenous breeds and because of the larger per-flock sample size used in this study (sixteen).

A survey of antibodies to pestivirus in England and Wales found $10.8 \%$ of individual animals and $37.4 \%$ of flocks seropositive (Sands and Harkness, 1978). The most recent European work suggests an individual animal seroprevalence of $8.3 \%$ among Danish sheep (Tegtmeier et al., 2000). In Switzerland, the influence of flock management and sheep breed was clearly illustrated (Schaller et al., 2000) as $20 \%$ of pedigree flocks were infected compared to $65 \%$ of other flock types. Five of six French flocks showed positive serology (Brugere-Picoux, 1987), while an animal seroprevalence of $17.9 \%$ was reported in Spanish sheep (Mainar-Jaime and Vazquez-Boland, 1999). A pestivirus seroprevalence level of $4.5 \%$ among individual animals was documented in Norwegian sheep with $18 \%$ of sheep flocks showing exposure to the virus (Loken et al., 1991). Seroprevalences of $25 \%$ and $66 \%$ to pestivirus were reported in two Irish flocks with recent histories of clinical border disease (Hamilton and Timoney, 1973).

There was considerable geographical variation in levels of flock seroprevalence. Higher numbers of infected flocks were in the east and midlands, with many apparently uninfected flocks being found in the south and west of the country. The influence of stocking density and farm management practices on regional variation in pestivirus antibody prevalence has been discussed previously (Sands and Harkness, 1978; Lamontagne and Roy, 1984; Loken et al., 1991; Graham et al., 2001). Figures 1 and 2 illustrate these geographical differences and suggest that the highest pestivirus flock seroprevalences are in the central lowland counties of Ireland. Higher stocking densities and husbandry systems associated with more intensive sheep production may explain these higher levels of pestivirus seroconversion. Substantial regional variations in the use of particular sheep breeds and variable breed susceptibility to pestivirus may confound these management effects (Schaller et al., 2000).

All flock disease problems were considered significant during the state of high alert of the foot-and-mouth disease crisis, so it may be assumed that the vast majority of seropositive sheep did not have clinical signs of oral/digital lesions, pyrexia, or depression. Similarly, abortion storms were reportable events during this period, and none was reported in the 91 flocks sampled.

In the current study, the sample collection period was early to mid-summer, some months after the normal lambing season. As pestiviruses are commonly released in foetal fluids at lambing, high seroprevalence levels would be expected when sampling at this time (Nettleton et al., 1998). Beyond the lambing season, the virus seems to spread rather slowly among sheep at grass. Only four out of 22 sheep seroconverted after three months of mixing with known persistently-infected animals (Bonniwell et al., 1987). Trough-feeding, housing and other intensive farm management features are considered to increase the pestivirus transmission rates among sheep (Nettleton et al., 1998).

More targeted testing of antibody-positive flocks was undertaken to evaluate disease dynamics within such flocks. None of these seropositive flocks had levels of infection above $30 \%$, and the mean level was less than $25 \%$. Cattle were present on all of these higher incidence farms. Levels of seroprevalence of this order would suggest medium-level pestivirus transmission, probably due to the presence of persistentlyinfected animals within these flocks or transmission from cattle to sheep on these farms.

Persistently-infected animals can show quite marked temporal changes in levels of pestivirus antibodies (Roeder et al., 1987). There has been little research as to the probability of one or more animals, persistently infected with border disease virus, acting as the source of above-average infection rates in 
particular sheep flocks, although a comparable situation exists with BVDV in cattle (Carlsson and Belak, 1994). Examination of the ELISA-positive sera by comparative serum neutralisation tests found that the highest antibody titres were to the NADL strain of BVDV. Similar findings were obtained in the NI sheep and it is suggested that interspecies pestiviral transmission in Ireland may be predominantly from cattle to sheep, mainly due to BVDV (Graham et al., 2001). This is supported by the detection of four-fold or greater antibody titres to BVDV than to BDV strain $137 / 4$, in over $80 \%$ of samples tested and, similarly, fourfold or greater antibody titres to BVDV than to BDV strain Moredun, in almost $40 \%$ of samples tested.

Cattle were present on a very high proportion $(92 \%)$ of the farms tested, so this study proved unsuitable to test fully the relationship between the concurrent presence of cattle and levels of ovine pestivirus seroconversion. Interestingly, only $2.7 \%$ of samples collected from sheep farms where cattle were not present, were pestivirus antibody-positive, in contrast to $5.8 \%$ seropositive on farms where cattle were present. Transmission of BVDV from cattle to sheep has been reported before (Carlsson, 1991) but pestiviruses are not thought to spread commonly from sheep to cattle (Paton et al., 1999; Pratelli et al., 2001).

The high serum neutralising antibody titres to the Moredun strain of BDV detected in the present study could be due to cross reactions with BVDV but may also indicate the local occurrence of BDV strains related to subtype B (Vilcek et al., 1997). Three sera had four-fold or greater antibody titres to BDV strain Moredun than to BVDV. Low antibody titres to BDV strain $137 / 4$ in both the Republic of Ireland and Northern Ireland (Graham et al., 2001) suggest that BDV strains of subtype A may not be present in Ireland. While infection of pigs in Ireland with ruminant pestiviruses is uncommon (O'Connor et al., 1991; Graham et al., 2001), the strains circulating in sheep have the potential to cross-react in serological surveillance and diagnostics for CSFV in pigs in Ireland, as well as mimicking chronic or pre-natal CSFV infection clinically (Terpstra and Wensvoort, 1988; Paton and Done, 1994).

This study highlights the apparently uneven distribution of pestivirus infection among Irish sheep, with some flocks apparently naïve to the viruses involved and, as a result, highly vulnerable to outbreaks of pestivirus-related disease.

\section{Acknowledgements}

The authors thank Dr R. Riebe, Federal Research Institute of Virus Diseases of Animals, Isle of Riems, Germany, for provision of the SFT-R cell line and B. Wilson, P. Dillon and V. Geraghty for providing FBK cultures and the NADL strain of BVDV.

\section{References}

Adair, B.M., McFerran, J.B., McKillop, E.R. and McCullough, S.J. (1984). Survey for antibodies to respiratory viruses in two groups of sheep in Northern Ireland. Veterinary Record 115: 403-406.
Bonniwell, M.A., Nettleton, P.F., Gardiner, A.C., Barlow, R.M. and Gilmour, J.S. (1987). Border disease without nervous signs or fleece changes. Veterinary Record 120: 246-249.

Brugere-Picoux, J. (1987). Border disease in France. Veterinary Record 120: $374-374$

Carlsson, U. (1991). Border disease in sheep caused by transmission of virus from cattle persistently infected with bovine virus diarrhoea virus. Veterinary Record 128: 145-147.

Carlsson, U. and Belak, K. (1994). Border disease virus transmitted to sheep and cattle by a persistently infected ewe: epidemiology and control. Acta Veterinaria Scandinavica 35: 79-88.

Costelloe, J.A., Gaynor, M.C., Gaynor, S., McAteer, W.J. and O'Reilly, P.J. (2002). Control of foot-and-mouth disease: lessons from the experience of Ireland. International Office of Epizootics Scientific and Technical Review 21: 739-750.

Graham, D.A., Calvert, V., German, A. and McCullough, S.J. (2001). Pestiviral infections in sheep and pigs in Northern Ireland. Veterinary Record 148: 69-71.

Gunn, M. (1996). BVD in cattle: Continuing education. Irish Veterinary Journal 49: 434-435.

Hamilton, A.F. and Timoney, P.J. (1973). Bovine virus diarrhoeamucosal disease virus and border disease. Research in Veterinary Science 15: 265-267.

Huck, R.A., Evans, D.H., Woods, D.G., King, A.A. and Stuart, P. (1975). Border disease of sheep. Comparison of the results of serological testing using complement fixation, immunodiffusion, neutralisation and immunofluorescent techniques. British Veterinary Journal 131: 427-435.

Hurtado, A., Garcia-Perez, A.L., Aduriz, G. and Juste, R.A. (2003). Genetic diversity of ruminant pestiviruses from Spain. Virus Research 92: 67-73.

Jeffrey, M. and Roeder, P.L. (1987). Variable nature of border disease on a single farm: clinical and pathological description of affected sheep. Research in Veterinary Science 43: 22-27.

Jensen, M.H. (1981). Detection of antibodies against hog cholera virus and bovine viral diarrhea virus in porcine serum. A comparative examination using CF, PLA and NPLA assays. Acta Veterinaria Scandinavica 22: 85-98.

Lamontagne, L. and Roy, R. (1984). Presence of antibodies to bovine viral diarrhea-mucosal disease virus (border disease) in sheep and goat flocks in Quebec. Canadian Journal of Comparative Medicine 48: 225-227

Lenihan, P. and Collery, P. (1977). Bovine viral diarrhoea infection in pigs in Ireland: a serological survey and an epidemiological study. Hog cholera/Classical Swine Fever and African Swine Fever Publication ref. EUR 5904: 314-322.

Loken, T., Krogsrud, J. and Larsen, I.L. (1991). Pestivirus infections in Norway. Serological investigations in cattle, sheep and pigs. Acta Veterinaria Scandinavica 32: 27-34.

Lunden, A., Carlsson, U. and Naslund, K. (1992). Toxoplasmosis and border disease in 54 Swedish sheep flocks. Seroprevalence and incidence during one gestation period. Acta Veterinaria Scandinavica 33: 175-184. 
Mainar-Jaime, R.C. and Vazquez-Boland, J.A. (1999). Associations of veterinary services and farmer characteristics with the prevalences of brucellosis and border disease in small ruminants in Spain. Preventative Veterinary Medicine 40: 193-205.

Nettleton, P.F., Gilray, J.A., Russo, P. and Dlissi, E. (1998). Border disease of sheep and goats. Veterinary Research 29: 327-340.

O'Connor, M., Lenihan, P. and Dillon, P. (1991). Pestivirus antibodies in pigs in Ireland. Veterinary Record 129: 269.

Paton, D.J. and Done, S.H. (1994). Congenital infection of pigs with ruminant-type pestiviruses. Journal of Comparative Pathology 111 : 151-163.

Paton, D.J., Sharp, G. and Ibata, G. (1999). Foetal cross-protection experiments between type 1 and type 2 bovine viral diarrhoea virus in pregnant ewes. Veterinary Microbiology 64: 185-196.

Pearson, J.E. (1992). Hog cholera diagnostic techniques. Comparative Immunology, Microbiology and Infectious Diseases 15: 213-219.

Pratelli, A., Bollo, E., Martella, V., Guarda, F., Chiocco, D. and Buonavoglia, C. (1999). Pestivirus infection in small ruminants: virological and histopathological findings. New Microbiology 22: 351-356.

Pratelli, A., Martella, V., Cirone, F., Buonavoglia, D., Elia, G., Tempesta, M. and Buonavoglia, C. (2001). Genomic characterization of pestiviruses isolated from lambs and kids in southern Italy. Journal of Virological Methods 94: 81-85.

Roeder, P.L., Jeffrey, M. and Drew, T.W. (1987). Variable nature of border disease on a single farm: the infection status of affected sheep. Research in Veterinary Science 43: 28-33.

Sands, J.J. and Harkness, J.W. (1978). The distribution of antibodies to Border disease virus among sheep in England and Wales. Research in Veterinary Science 25: 241-242.

Sawyer, M.M., Schore, C.E., Menzies, P.I. and Osburn, B.I. (1986). Border disease in a flock of sheep: epidemiologic, laboratory, and clinical findings. Journal of American Veterinary Medical Association 189: 61-65.

Schaller, P., Vogt, H.R., Strasser, M., Nettleton, P.F., Peterhans, E. and Zanoni, R. (2000). Seroprevalence of maedi-visna and border disease in Switzerland. Schweizer Arciv für Tierheilkunde 142: 145153.

Tegtmeier, C., Stryhn, H., Uttentha, I., Kjeldsen, A.M. and Nielsen, T.K. (2000). Seroprevalence of border disease in Danish sheep and goat herds. Acta Veterinaria Scandinavica 41: 339-344.

Terpstra, C. and Wensvoort, G. (1988). Natural infections of pigs with bovine viral diarrhoea virus associated with signs resembling swine fever. Research in Veterinary Science 45: 137-142.

Vilcek, S., Drew, T.W., McGoldrick, A. and Paton, D.J. (1999). Genetic typing of bovine pestiviruses from England and Wales. Veterinary Microbiology 69: 227-237.

Vilcek, S., Nettleton, P.F., Paton, D.J. and Belak, S. (1997). Molecular characterization of ovine pestiviruses. Journal of General Virology 78: 725-735. 